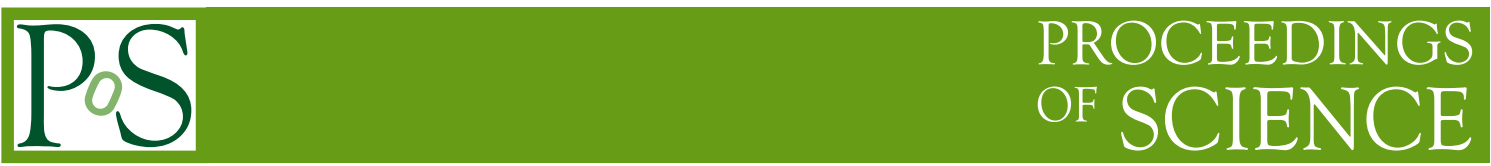

\title{
CALET's Sensitivity to Dark Matter and Astrophysical Sources
}

\author{
Holger Motz* for the CALET collaboration \\ Waseda University \\ E-mail: motzeaoni.waseda.jp
}

The Calorimetric Electron Telescope (CALET) is scheduled to be launched to the ISS before October of this year and will measure the energy and direction distribution of electron+positron cosmic rays well into the $\mathrm{TeV}$ range during a 5-year mission. With a $1: 10^{5}$ proton rejection rate and an energy resolution of $2 \%$, it is capable of detecting even small features in the spectrum. Combining the measurement of the total electron and positron flux by CALET with the positron fraction data from the AMS-02 experiment, it will be possible to significantly constrain models of Dark Matter annihilating in the galactic halo. Assuming the positron excess is caused by an astrophysical single power law source emitting an equal amount of electrons and positrons, the expected Dark Matter limits for 5-yr are presented, based on simulated data for CALET together with the recent positron fraction of AMS-02. These predictions are compared to limits derived with the same procedure using current experimental data. While this power law source explaining the positron excess could be a single nearby pulsar, the large number of pulsars discovered by radio and $\mathrm{X}$-ray telescopes makes an overlapping spectrum from several strongly contributing sources likely. Based on a numerical cosmic ray propagation simulation, the cosmic-ray spectra from nearby pulsar wind nebulae have been calculated and the expected capability of CALET to discern the multiple overlapping spectra, with parameters chosen to explain the observed cosmic ray excess, from the single power law spectrum of one pulsar is shown.

The 34th International Cosmic Ray Conference,

30 July- 6 August, 2015

The Hague, The Netherlands

${ }^{*}$ Speaker. 


\section{Introduction}

The excess in the positron fraction measured by the AMS-02 collaboration [1] is proposed by them to originate from a single power law extra source with an exponential cut-off, emitting an equal flux of electrons and positrons. To investigate the sensitivity of CALET [2] to Dark Matter annihilation signatures, the parametrization introduced by this approach is extended to the total electron+positron flux and to include propagation effects at higher energies. Under the assumption that the extra component is purely from a nearby pulsar, the parametrization together with the AMS-02 total electron+positron flux up to one $\mathrm{TeV}$ [3] is used to extrapolate this scenario into the $\mathrm{TeV}$ region. The parametrization and parameter choices are corroborated by numerical propagation calculations. Based on this prediction, the expected CALET total flux measurement results for 5 years of data-taking were simulated. Adding a hypothetical additional component from Dark Matter, the limits that could be set, subject to CALET data matching the pure pulsar case, on annihilation of several Dark Matter candidates have been calculated. CALET will provide the first precise measurement of the $\mathrm{TeV}$ region, with the main objective to identify signatures of nearby supernova remnants [4], making a different outcome probable. Adressing this, the capability of CALET to discriminate between the single pulsar case and the flux from multiple astrophysical sources was calculated based on the same parametrization.

\section{Dark Matter Sensitivity of CALET}

\subsection{Method of Dark Matter Sensitivity Calculation}

To describe the local cosmic ray electron and positron spectrum with a primary component originating from distant supernovae and a secondary component from nuclei cosmic rays interacting with the interstellar medium in a parametrization, two power law indices $\gamma_{e}$ and $\gamma_{e^{+}}$and two coefficients $C_{e}$ and $C_{e^{+}}$for the combined electron+positron and positron-only flux are required. Radiative energy losses of this diffuse background spectrum are modeled as an exponential cut-off at around energy $E_{\text {cut }_{d}}$. The scenario of a single pulsar as extra source is parametrized by a power law term for both electrons and positrons with index $\gamma_{s}$, coefficient $C_{s}$ and cut-off energy $E_{\text {cut }_{s}}$. For the purpose of studying CALET's sensitivity to it, a component from Dark Matter annihilation $\Phi_{D M}$ scaled by a Boost Factor $B F$ is introduced, so that the total flux is given by:

$$
\Phi_{e}(E)=2 \Phi_{D M}(E) \cdot B F+C_{e} E^{\gamma_{e}}\left(2 \frac{C_{s}}{C_{e}} E^{\gamma_{s}-\gamma_{e}} \cdot \exp \left(\frac{-E}{E_{\text {cut }_{s}}}\right)+\left(\frac{C_{e^{+}}}{C_{e}} \cdot E^{\gamma_{e}+-\gamma_{e}}+1\right) \cdot \exp \left(\frac{-E}{E_{\text {cut }_{d}}}\right)\right)
$$

With both Dark Matter annihilation and the pulsar emitting an equal ratio of electrons and positrons, the positron fraction is calculated as:

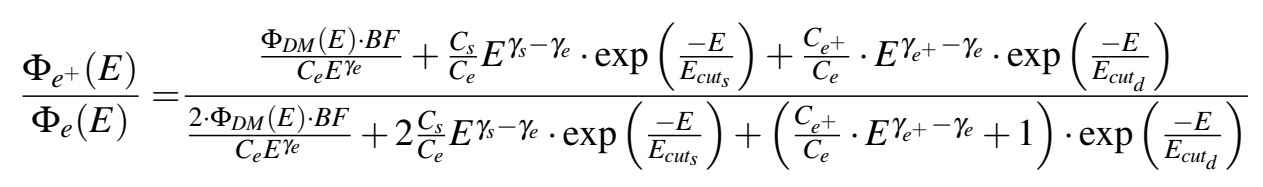

The independent parameters $B F, C_{e}, \gamma_{e}, \frac{C_{s}}{C_{e}}, \gamma_{s}-\gamma_{e}, E_{c u t_{s}}, \frac{C_{e^{+}}}{C_{e}}, \gamma_{e^{+}}-\gamma_{e}, E_{\text {cut }_{d}}$ define this model, together with $\Phi_{D M}(E)$ which depends on the Dark Matter candidate and is calculated using DarkSUSY [5] assuming NFW profile [6] and a local Dark Matter density $\rho_{0}=0.3 \mathrm{GeV} \mathrm{cm}^{-3}$. 

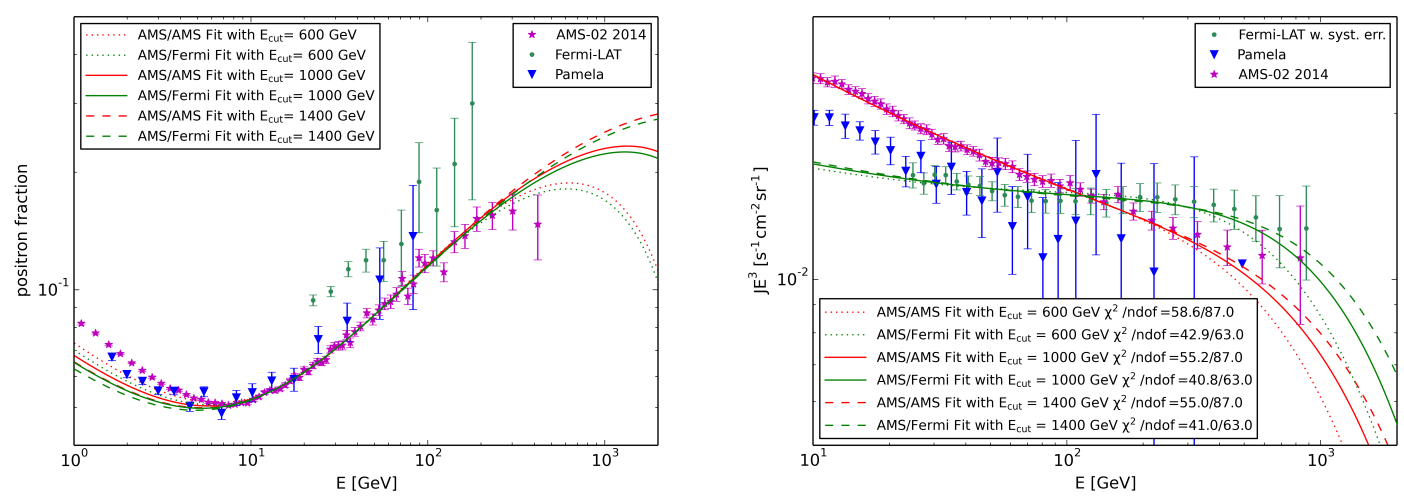

Figure 1: Best fit results for the single pulsar extra source to AMS-02 positron fraction data in combination with AMS-02 total flux data (AMS/AMS-Fit) and Fermi-LAT total flux data (AMS/Fermi-Fit). As shown in the legend, the extra source cut-off energy $E_{c u t}$ does not significantly change $\chi^{2}$, and therefore cannot be well determined by the fit for either case. $\gamma_{e^{+}}-\gamma_{e}$ is set to -0.4 .

In order to determine the background models for the sensitivity calculation, a combined fit of formula 2.2 to the AMS-02 positron fraction, and formula 2.1 to the AMS-02 or Fermi-LAT total flux [7] data is done. Because charge-dependent solar modulation changes the measured flux and positron fraction below $10 \mathrm{GeV}$ [8], only data-points above $10 \mathrm{GeV}$ were used in the fit. The cutoff energy $E_{\text {cut }_{d}}$ cannot be determined well by the fit, since it has influence only at high energy. It was fixed to $2 \mathrm{TeV}$ and the applicability of the exponential term confirmed by numerical simulation results (described in 2.2). The fit quality also changes only marginally with $E_{c u t_{s}}$. This is shown in Figure 1 for three discrete values $(0.6 \mathrm{TeV}, 1.0 \mathrm{TeV}$ and $1.4 \mathrm{TeV})$, which are accordingly used as independent background cases in the sensitivity calculations. The parameter $\gamma_{e^{+}}-\gamma_{e}$, which describes the slope of the positron fraction without additional source, has only significant influence below $10 \mathrm{GeV}$, making it also unobtainable by the fit. With the secondary positrons originating from interaction of primary protons with the interstellar medium, $\gamma_{e^{+}}-\gamma_{e}$ is close to $-\delta$, the exponent determining the diffusion coefficient's energy dependence. By this relation, its range is constrained -0.3 to -0.7 , and the discrete values $-0.3,-0.4,-0.5,-0.6$ and -0.7 were studied independently.

As starting point for the Dark Matter sensitivity calculation serve the fits of the single pulsar case without the Dark Matter term to AMS-02 data shown in figure 1. To these background cases, the Dark Matter term is added both in total flux and positron fraction, and $B F$ increased in steps until the resultant $\chi^{2}$ is larger than the critical 95\%CL $\chi^{2}$ value for the number of free parameters in the fit. After each increase of $B F$, all free parameters of the parametrization are optimized again to adapt them to the newly added Dark Matter component. Contrary to the fit of the background case, $E_{\text {cut }}$ is also treated as a free parameter. Since not a true and unique $\chi^{2}$ minimum, but any set of parameters with $\chi^{2}$ below $95 \%$ CL confirms that the assumed value of $B F$ is not excluded, the "Migrad" and "Simplex" algorithms of Minuit are used in series, which avoids aborting the fit due to non-unique minima. However, $\chi^{2}$ should steadily increase with $B F$ to obtain a conservative limit. Thus it is checked that $\chi^{2}$ of the final fit is within 95\%CL threshold, with the datapoint discarded if not. The limits on $B F$ obtained by this method are translated into limits on speed- 

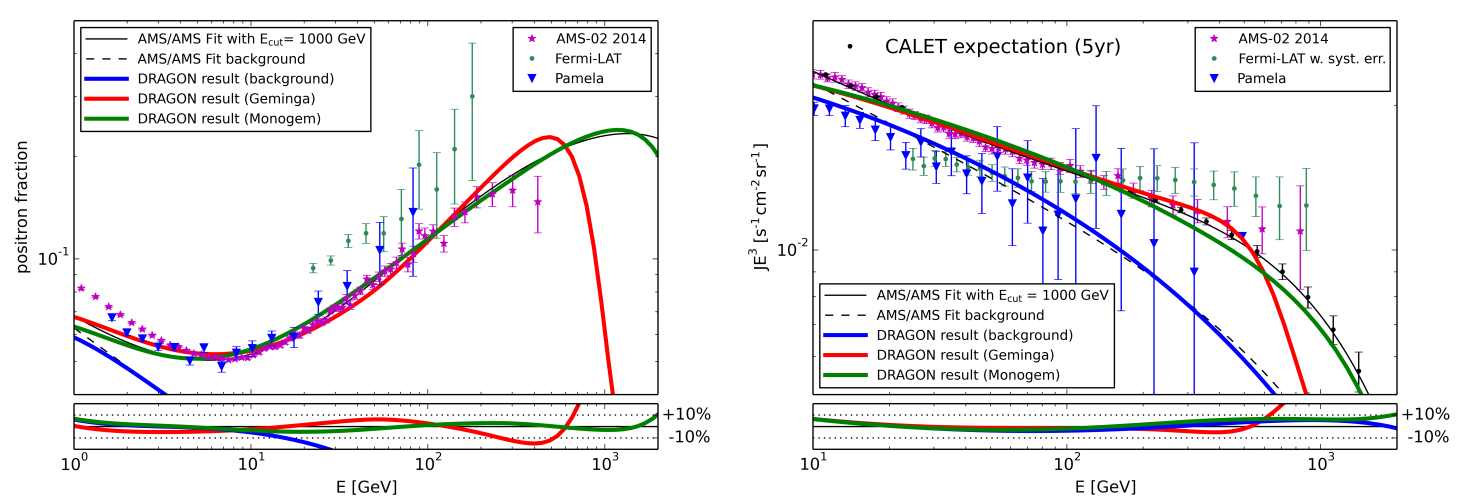

Figure 2: Best fit results for the single pulsar extra source $\left(E_{\text {cut }}=1 \mathrm{TeV}\right)$ to AMS-02 positron fraction data and AMS-02 total flux data compared to the results of the DRAGON program. In the lower panel the fractional difference (Parametrization-DRAGON)/Parametrization is plotted, showing that in the most relevant energy ranges the agreement is better than $10 \%$.

averaged $\mathrm{x}$-section $\langle\sigma v\rangle$ by multiplication with $\langle\sigma v\rangle=3 \cdot 10^{-26} \mathrm{~cm}^{3} \mathrm{~s}^{-1}$, the value for which the Dark Matter flux $\Phi_{D M}$ is calculated. To simulate CALET data, statistical fluctuations to the expected event rates were taken into account by generating 100 event samples for the assumed single pulsar case. Event energies are randomly generated with the distribution of the predicted signal for 5 years of data-taking, which assumes an aperture of $1200 \mathrm{~cm}^{2} \cdot \mathrm{sr}$ for CALET [9] and a reconstruction efficiency of $90 \%$. The events are then filled into 20 bins per decade for a total of 60 bins from $10 \mathrm{GeV}$ to $10 \mathrm{TeV}$, which was estimated to be suitable for the applied $\chi^{2}$ analysis. The expected limits are determined by performing the above described fitting procedure for each sample, and taking the average $B F$ value. The limits for current data are obtained by using AMS-02 total flux measurement instead of simulated CALET data.

\subsection{Comparison of the Parametrization to Propagation Simulation Results}

To ensure that the adopted parametrization is in accordance with current models of cosmic ray propagation, simulations with DRAGON [10] were done. DRAGON was chosen instead of GALPROP [11], because it features a non-equidistant spatial grid and furthermore allowed the primary electron source distribution to be modeled according to the galaxy's spiral arm structure described in [12]. The injection spectrum index for electrons $\gamma_{i_{e}}$ is choosen equal to the index for nucleons $\gamma_{i_{n}}$ by default, which implies $\gamma_{e^{+}}-\gamma_{e}$ equal to $-\delta$. To reproduce the proton spectrum measured by PAMELA [13], $\gamma_{i_{n}}=-2.8+\delta$ is required, where $\delta$ is the exponent in the diffusion coefficient's energy relation, $D=D_{0} \cdot\left(E / E_{0}\right)^{\delta}$, with $E_{0}=4 \mathrm{GeV}$. For $D_{0}$, values from $2.7 \cdot 10^{28} \mathrm{~cm}^{2} / \mathrm{s}$ to $7.2 \cdot 10^{28} \mathrm{~cm}^{2} / \mathrm{s}$ were considered. Under these conditions, good agreement for the background as shown in Figure 2 between DRAGON and the parametrization fit to AMS-02 data equally in positron fraction and total flux is only given for $\gamma_{e^{+}}-\gamma_{e}=-\delta=-0.4\left(D_{0}=6.2 \cdot 10^{28} \mathrm{~cm}^{2} / \mathrm{s}\right)$, which was therefore chosen as the default case. The exponential cut-off due to energy loss in the parametrization is confirmed, with best agreement if the parameter $E_{\text {cut }_{d}}$ is set to $2 \mathrm{TeV}$.

The astrophysical extra source case was implemented by simulating the Geminga pulsar wind nebula (PWN) at a distance of $0.25 \mathrm{kpc}$ and with an age of $342 \mathrm{kyr}$ and the Monogem PWN at 


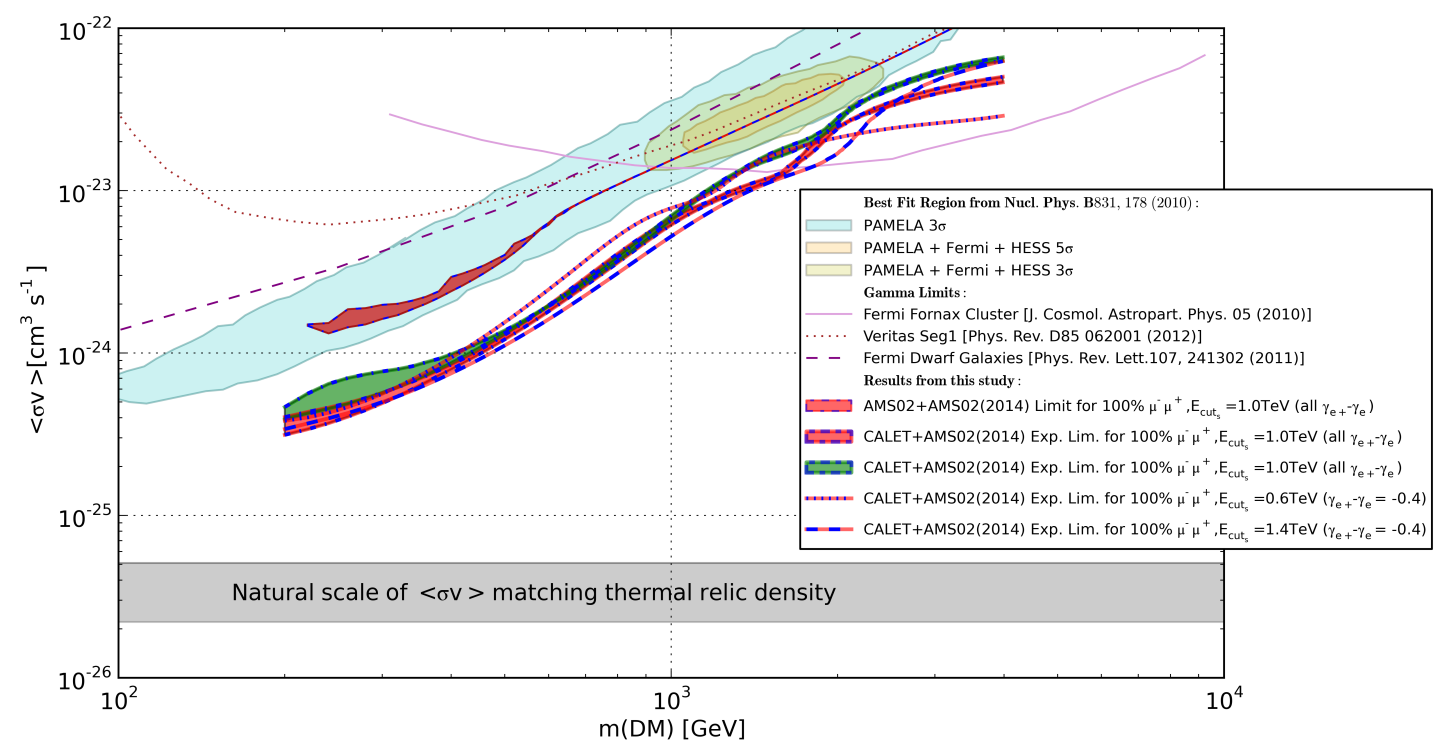

Figure 3: Expected Dark Matter annihilation limits (100\% $\mu \bar{\mu}$-channel) from CALET for different values of $\gamma_{e^{+}}-\gamma_{e}$ and $E_{c u t_{s}}$. The expected limit plotted with a red filled area shows the range for varying $\gamma_{e^{+}}-\gamma_{e}$ from -0.3 to -0.7 in the AMS/AMS-Fit background case, the one with a green filled area is the same for the AMS/Fermi case. The "Best Fit Region" corresponds to the parameters expected if the positron excess is caused only by Dark Matter annihilation.

a distance of $0.28 \mathrm{kpc}$ and an age of $86 \mathrm{kpc}$, using the information from the ATNF catalog [14] (details of the PWN cosmic ray injection are given in section 3). From a scan over the injection power law index $\gamma_{i_{s}}$ in steps of 0.1 and cut-off energy $E_{c u t_{i}}$ at values $1 \mathrm{TeV}, 3 \mathrm{TeV}$ and $10 \mathrm{TeV}$, best agreement is given for $\gamma_{i_{s}}=2.1$ and $E_{c u t_{i}}=10 \mathrm{TeV}$ for Geminga and $\gamma_{i_{s}}=2.3$ and $E_{c u t_{i}}=3 \mathrm{TeV}$ for Monogem. For these calculcations, the galaxy within in a region of $32 \times 32 \times 12 \mathrm{kpc}$ was simulated on a non-equidistant, three-dimensional grid with a basic grid size of $0.5 \mathrm{kpc}$, which is reduced in steps to $0.01 \mathrm{kpc}$ around the pulsars and the solar system. While the spectrum of the older Geminga pulsar shows a cut-off from radiative energy loss upward of $500 \mathrm{GeV}$, is the Monogem spectrum in full agreement with the parametrization assuming $E_{\text {cuts }}=1 \mathrm{TeV}$, demonstrating that though the spectrum in the $\mathrm{TeV}$ region is unknown yet, the parametrized single pulsar case is a viable scenario.

\subsection{Expected Limits on Dark Matter Annihilation from CALET Data}

In Figure 3, the current AMS-02 limit and predicted AMS-02 + CALET limits under different assumptions for the $100 \% \mu \bar{\mu}$-channel are shown. The expected limits have no significant dependence on $\gamma_{e^{+}}-\gamma_{e}$, but their shape depends on the initial value of $E_{c u t_{s}}$ as it influences for which mass the Dark Matter spectrum is closest to the assumed pulsar spectrum. Choosing the AMS/Fermi background case results as well in a slightly different, but comparable predicted limit. All these limits would surpass those of indirect Dark Matter search in the $\gamma$-ray spectra of extragalactic sources and exclude muon-channel annihilation of Dark Matter as the origin of the positron 


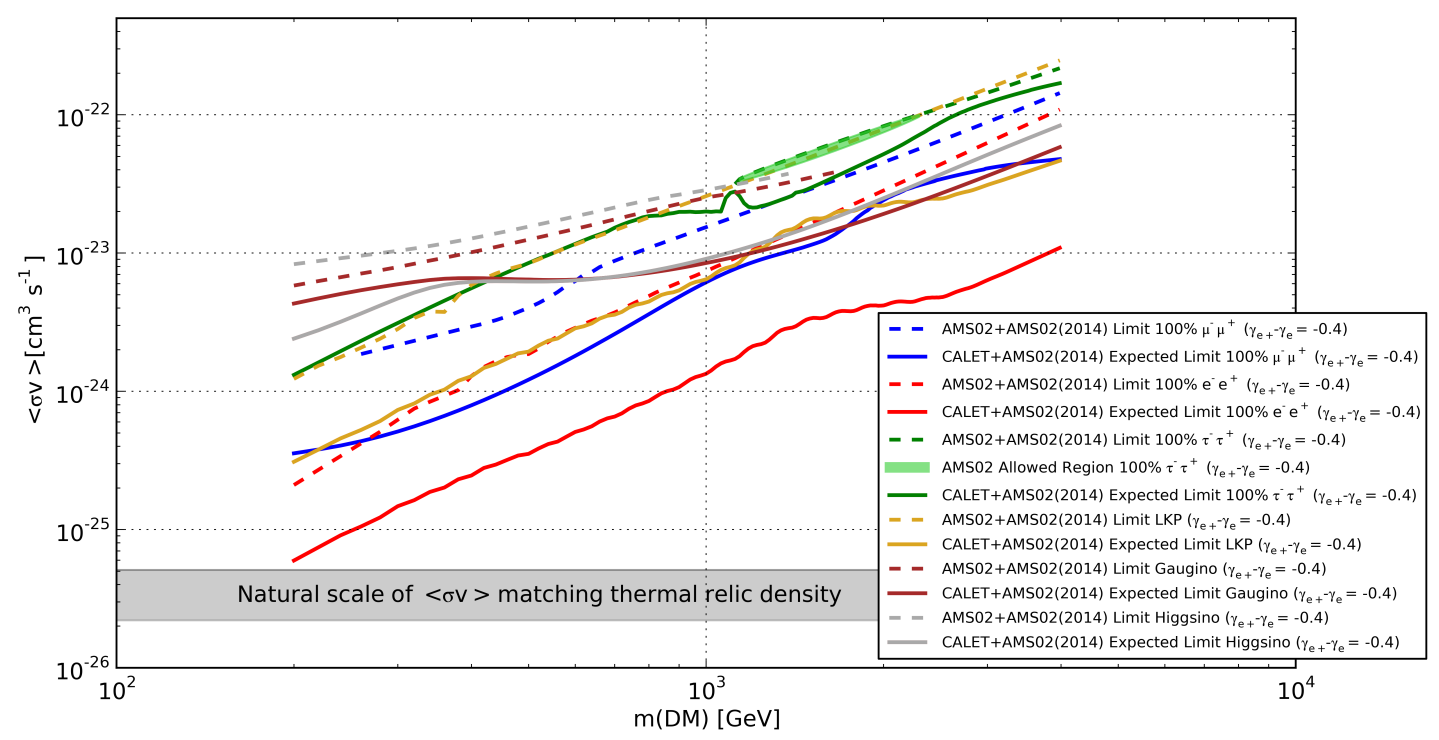

Figure 4: Current (AMS-02) and expected (AMS-02 + CALET) annihilation $x$-section limits for different Dark Matter candidates, assuming $E_{\text {cut }}=1 \mathrm{TeV}$. For $\tau^{+} \tau^{-}$-annihilating Dark Matter, current AMS-02 data shows an "Allowed Region" where the excess is caused only by Dark Matter annihilation. Since they fail the $\chi^{2}$-test for the final fit described in 2.1, no current AMS-02 limits exist for high mass Gaugino and Higgsino, as well as low mass of $\tau^{+} \tau^{-}$-annihilating Dark Matter.

excess. Figure 4 gives an overview of the expected limits for Dark Matter annihilation purely into leptons, as well as the LKP (Lightest Kaluza-Klein Particle), Gaugino and Higgsino Dark Matter candidates, which are defined by their annihilation branching ratios:

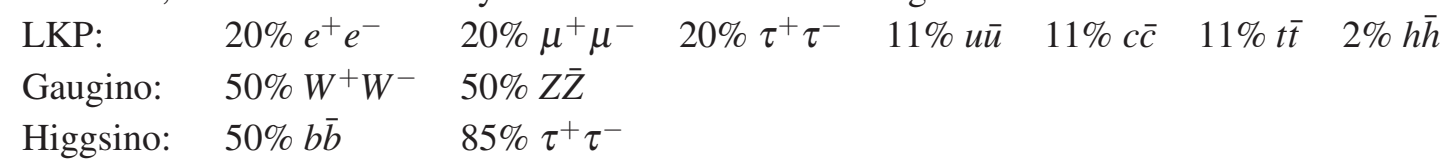

It is shown that by combination of CALET data with AMS-02 positron fraction, the limits on Dark Matter annihilation can be improved for all studied Dark Matter candidates, which is foremost attributed to better statistis of the CALET total flux data over the whole energy range. The similiarity of the $\tau^{+} \tau^{-}$-channel's spectrum to the pulsar spectrum (current AMS-02 data allows the positron excess to be fully attributed to it) results in a generally weak limit with a dent just above $1 \mathrm{TeV}$, where the combination of Dark Matter annihilation and a modified pulsar spectrum matches the assumed spectrum best. For LKP and annihilation to $e^{+} e^{-}$the improvement is approximately half an order of magnitude. This is due to these cases featuring a sudden drop of the spectrum at the energy matching the mass of the Dark Matter particles, which causes a mismatch with the single pulsar assumption, since in contrast to AMS-02 data, the CALET data extends up to this energy with good statistics and energy resolution. 

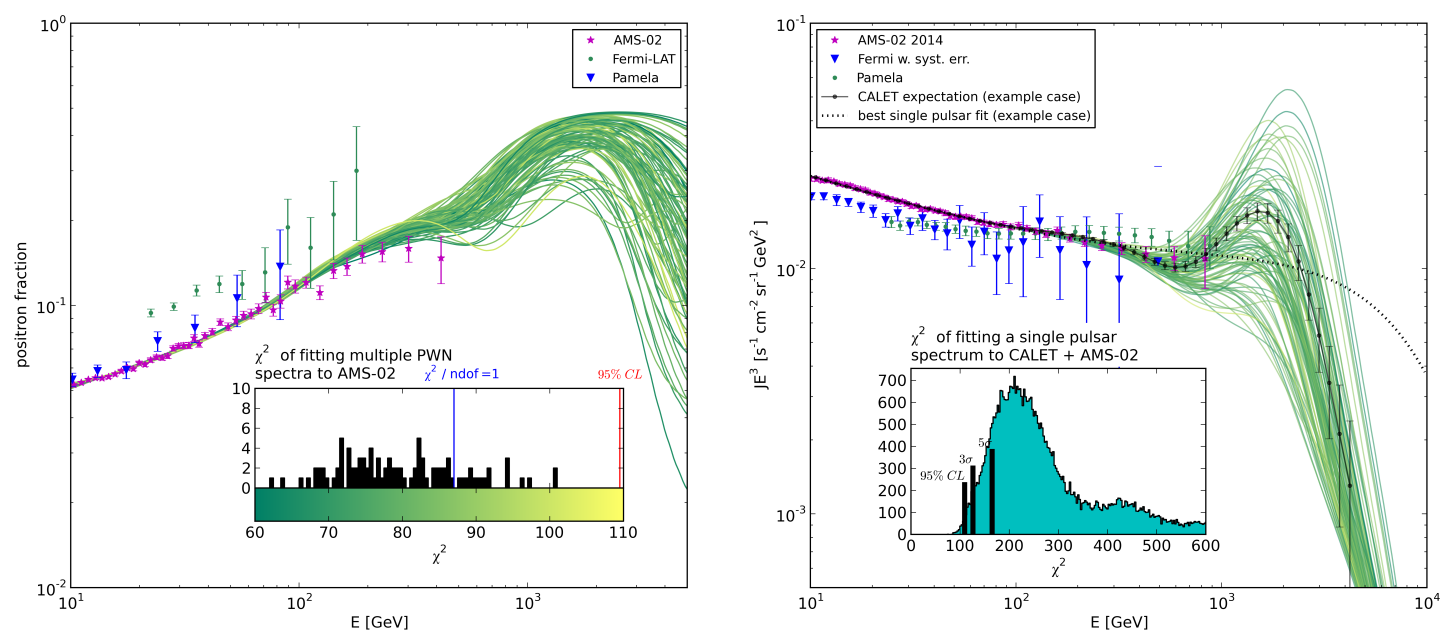

Figure 5: Positron fraction (left) and total flux (right) of the 100 simulated cases. The left embedded plot shows the initial $\chi^{2}$ distribution of fitting the cases to AMS-02, the right embedded plot shows that in the $\chi^{2}$ distribution of fitting the $100 \times 500$ samples of CALET data with a single power law a vast majority of samples is above the $95 \%$ CL limit and thus can be distinguished from the single pulsar case. The expected statistics of CALET for one example case and the best fit by a single pulsar spectrum are shown in black.

\section{Multiple Pulsar Spectrum in the TeV-Region}

CALET's measurement of the $\mathrm{TeV}$ region could reveal deviations from the single pulsar case well describing current experimental results, since a large number of pulsars in the vicinity of the solar system are known, varying in distance and age, all likely contributing to the extra component flux. Unlike initial rotation energy and age of a pulsar, it is not possible to determine its cosmic ray injection characteristics from observation. To investigate the capability of CALET to identify a composite spectrum from the nearby known pulsars, the spectra from the 40 pulsars in the ATNF catalog [14] within $2 \mathrm{kpc}$ distance and younger than $1 \mathrm{Myr}$ were simulated with DRAGON for a range of injection indices from -1.0 to $-2.0(-1.0,-1.3,-1.5,-1,7,-2.0)$ and cut-off energies from 0.7 TeV to $10 \mathrm{TeV}(0.7 \mathrm{TeV}, 1 \mathrm{TeV}, 1.4 \mathrm{TeV}, 2 \mathrm{TeV}, 3 \mathrm{TeV}, 5 \mathrm{TeV}, 7 \mathrm{TeV}, 10 \mathrm{TeV})$. Following [15] and [16], the accelerated particles are assumed to be initially trapped in a pulsar wind nebula (PWN) and accumulated over the lifetime of the PWN, for which three values (10 kyr,20 kyr,40 kyr) were considered. When the PWN dissolves, they are released with the intensity attenuating exponentially, the decay constant being $10 \mathrm{kyr}$. The simulated spectra were combined in a random walk assigning the parameters above, as well as an efficiency factor corresponding to the fraction of energy converted into cosmic rays, to each PWN, in order to create 100 cases matching AMS-02 data. For each of these cases, 500 statistical samples of 5-year CALET data were simulated by the procedure described in 2.1 and used to determine the $\chi^{2}$ distribution of trying to fit a single source power law spectrum to them. It is shown by the combined $\chi^{2}$ distribution in Figure 5, that the single pulsar hypothesis could generally be rejected, with the exception of unlikely cases in which the overlap of multiple sources forms a spectrum similar to a single source by chance. 


\section{Conclusion}

CALET will measure the total electron+positron flux up to $20 \mathrm{TeV}$ with good statistics and energy resolution even in the $\mathrm{TeV}$ region. Assuming the positron excess is caused by a single nearby pulsar, the limits obtainable from CALET's measurement on Dark Matter annihilation purely into leptons, as well as for LKP, Gaugino and Higgsino Dark Matter with mass from $200 \mathrm{GeV}$ to $4 \mathrm{TeV}$ have been calculated. Their reliability with regard to nuisance parameters, and the agreement of the used parametrization with numerical propagation calculation were confirmed. The alternative scenario of emission from multiple pulsar wind nebulae causing the positron excess and dominating the TeV-region spectrum was studied as well, concluding that:

- If CALET data is found to match the single pulsar scenario, it will be possible to set significantly more stringent limits on Dark Matter annihilation compared to current experimental data, especially for annihilation to $e^{+} e^{-}$and LKP.

- Should CALET results not be compatible with the assumed single pulsar case in the TeVregion, the alternative explanation of an overlapping spectrum from multiple PWN can be tested and well discerned from the single source case.

CALET is optimized to detect nearby supernova remnants [4], making it possible to include them in the background model for Dark Matter search when CALET data becomes available. While the presented sensitivity assumes the positron excess is not caused by Dark Matter at all, the actual CALET data may hint at Dark Matter partially contributing to the positron excess or even causing it completely, for example in the form of decaying Dark Matter [17], in which case CALET data will be analyzed for discovering the signatures of these scenarios.

\section{References}

[1] AMS Collaboration, L. Accardo et al. Phys. Rev. Lett. 113 (Sep, 2014) 121101.

[2] CALET Collaboration, S. Torii. Paper ID 430 in this conference.

[3] AMS Collaboration, M. Aguilar et al. Phys. Rev. Lett. 113 (Nov, 2014) 221102.

[4] S. Torii NIM A 630 (2011), no. 155 - 57. Proceedings of RICAP 2009.

[5] P. Gondolo, J. Edsjo, P. Ullio, L. Bergstrom, M. Schelke, et al. JCAP 0407 (2004) 008.

[6] J. F. Navarro, C. S. Frenk, and S. D. White Astrophys.J. 490 (1997) 493-508.

[7] Fermi LAT Collaboration, M. Ackermann et al. Phys.Rev. D82 (2010) 092004.

[8] L. Maccione Physical Review Letters 110 (Feb., 2013) 081101.

[9] Y. Akaike, K. Kasahara, and S. Torii in Proceedings of ICRC 2011, vol. 6, p. 371, 2011.

[10] D. Gaggero, L. Maccione, G. Di Bernardo, C. Evoli, and D. Grasso Phys.Rev.Lett. 111 (2013), no. 2 021102. DRAGON website: http://www.dragonproject.org.

[11] http://galprop.stanford.edu/.

[12] P. Blasi and E. Amato JCAP 1201 (2012) 011.

[13] PAMELA Collaboration, O. Adriani et al. Science 332 (Apr., 2011) 69-.

[14] R. N. Manchester, G. B. Hobbs, A. Teoh, and M. Hobbs Astron.J. 129 (2005) 1993.

[15] D. Malyshev, I. Cholis, and J. Gelfand Phys.Rev. D80 (2009) 063005.

[16] N. Kawanaka, K. Ioka, and M. M. Nojiri Astrophys.J. 710 (Feb., 2010) 958-963.

[17] M. Ibe, S. Matsumoto, S. Shirai, and T. T. Yanagida Phys.Lett. $B 741$ (2015) 134-137. 